\title{
Modified Reactive Strength Index in Adolescent Athletes Competing in Different Sports and Its Relationship with Force Production
}

\author{
Leonidas Petridis", Zsófia Tróznai, Gergely Pálinkás, Irina Kalabiska, Tamás Szabó \\ Sport Science and Diagnostic Laboratory, University of Physical Education, Budapest, Hungary \\ *Corresponding author: petridis.leonidas@mnsk.hu
}

\begin{abstract}
The modified reactive strength index (RSImod) is used as a measurement of reactive strength and explosiveness. The main purpose of the current study was to assess RSImod in young athletes competing in various sports and to examine the relationship between RSImod and eccentric and concentric force and vertical displacement. Two-way ANOVA showed a significant main effect for sport group, but not for age group. Soccer $(0.47 \pm 0.09 \mathrm{~m} / \mathrm{s})$ and basketball players $(0.49 \pm 0.1 \mathrm{~m} / \mathrm{s})$ had significantly higher values than handball players $(0.36 \pm 0.07 \mathrm{~m} / \mathrm{s})$ and rowers $(0.35 \pm 0.07 \mathrm{~m} / \mathrm{s})$. Among the independent variables, average eccentric force had the strongest relationship with RSImod. In conclusion, RSImod differs between athletes from different sports, but not between athletes from different age groups in the examined age period. Also, RSImod is strongly associated mainly with the average eccentric force and secondarily with the average concentric force during the countermovement jump.
\end{abstract}

Keywords: countermovement jump, power, junior athletes, eccentric and concentric force

Cite This Article: Leonidas Petridis, Zsófia Tróznai, Gergely Pálinkás, Irina Kalabiska, and Tamás Szabó, "Modified Reactive Strength Index in Adolescent Athletes Competing in Different Sports and Its Relationship with Force Production." American Journal of Sports Science and Medicine, vol. 5, no. 2 (2017): 21-26. doi: 10.12691/ajssm-5-2-2.

\section{Introduction}

Reactive Strength Index modified (RSImod) is an alternative measure of the reactive strength index (RSI) and is used to measure reactive strength and explosiveness [1]. In order to avoid drop jumps out of concern for injury risks during RSI calculation, RSImod involves a more common jump test, the countermovement jump (CMJ). RSImod is calculated by dividing jump height by time to takeoff during the countermovement jump [2].

Reliability studies with RSI [3,4] or with RSImod [2,5] have shown high reliability for both measures. Previously established moderate to large correlations of RSImod with the rate of force development (RFD) indicate that RSImod can be used to measure explosiveness in athletes $[5,6]$. In a recent study [7] the authors confirmed the validity of the RSImod as a measure of explosiveness, suggesting that RSImod incorporates both essential factors of explosiveness, namely force and speed.

Studies with RSImod so far have included adult athletes $[2,5,7]$ revealing differences between male and female athletes [5] or between different plyometric exercises [2]. Between the ages of 15 to 18 , young athletes at high competition levels quite often are exposed to considerable training loads designed to prepare them to compete at the adult level. The potential to develop neuromuscular performance throughout this age period, however, is still unknown.
Explosiveness is of major importance in many sports. Successful performance in ball games, such as soccer, basketball and handball require well developed skills in agility, change of direction, acceleration or jumping ability. On the other hand, rowing is an endurance type sport and usually jumping is not included in the training methodology of rowers. The question thus arises as to whether RSImod can differentiate between athletes of different athletic backgrounds. This could provide coaches and sport practitioners with easy-to-use and reliable measures for the assessment of explosiveness in their athletes.

Average force applied to the jumper's center of mass and the distance over which this force is applied have a direct effect on the vertical takeoff velocity, which in turn influences jump height [8]. Given that force production is closely related to explosiveness and power $[9,10]$, it is assumed that maximal and average force developed during a vertical jump will be correlated to RSImod. In vertical jump, larger force usually will result in larger jump height $[7,11,12]$, while well-developed strength is necessary to perform a rapid jumping movement, decreasing the time to takeoff and eventually altering in either case RSImod.

As a ratio, RSImod can increase by either an increase in jump height or a decrease in time to takeoff. Between two jumps of similar height, shorter time to takeoff would increase RSImod, or between two jumps of similar time to takeoff, higher jump height also would increase RSImod, suggesting in both cases greater explosive ability. 
The primary purpose of this study is to measure and compare RSImod in young athletes between the ages of 15-18 years and between different sport disciplines. A secondary purpose is to evaluate the relationship between RSImod and force production, both eccentric and concentric.

\section{Methods}

\subsection{Subjects}

$\mathrm{N}=115$ male athletes participated in this cross-sectional study and were divided into four age groups: 15 years old $(n=23) ; 16$ years old $(n=28) ; 17$ years old $(n=36)$; and 18 years old $(n=28)$. Age groups were formed based on decimal ages. Subjects participated in soccer $(n=58)$; basketball $(n=18)$; handball $(n=24)$ and rowing $(n=15)$ at an elite level in their respective age groups. All athletes attended training sessions on a regular basis for at least seven hours per week, and during the measurements all of them were in their regular in-season phase. Several days before the measurements all the athletes and their parents/guardians were informed about the types of measurements, and a written consent form was signed by the athletes $(>18$ years) or their parents/guardians $(<18$ years). Descriptive characteristics of the participants are presented in Table 1 and Table 2.

\subsection{Measurements}

The athletes arrived at the laboratory in the morning hours and underwent a cardiology, anthropometry and body composition examination. Anthropometric dimensions were collected according to the Martin method [13], body height was measured with an anthropometer to the nearest $0.1 \mathrm{~cm}$, and body weight was measured with a digital scale (Seca 888) to the nearest $0.01 \mathrm{~kg}$.

Vertical jumps were measured on a $0.6 \times 0.6 \mathrm{~m}$ force platform (model FP4, HUR Labs Oy, Tampere, Finland). A standard warm up routine was performed, including light aerobic and stretching exercises. Verbal instructions and a familiarization session were given for the CMJ. The athletes performed three jumps with one minute of passive rest between each attempt; the best jump was recorded for statistical analysis. All jumps were performed without an arm swing, with hands kept on hips. CMJ was performed from a standing position, and the athletes bent their knees to approximately 90 degrees and then immediately jumped as high as possible. All data were collected and analyzed with HUR Labs Force Platform Software Suite 2.40 (HUR Labs Oy, Tampere, Finland).

Variables extracted from the force-time data included: jump height; average eccentric $\left(\mathrm{F}_{\mathrm{ecc}}\right)$ and concentric force $\left(\mathrm{F}_{\text {con }}\right)$; time duration of the eccentric $\left(\mathrm{t}_{\mathrm{ecc}}\right)$ and the concentric phases $\left(t_{\text {con }}\right)$ and vertical displacement of the eccentric $\left(\mathrm{Vh}_{\mathrm{ecc}}\right)$ and concentric phases $\left(\mathrm{Vh}_{\mathrm{con}}\right)$ of the jump. Average eccentric force was calculated from the moment when ground reaction force exceeded body weight to the deepest point of the countermovement (velocity is zero), and average concentric force was calculated from the bottom of the countermovement to the moment of takeoff. $t_{\mathrm{ecc}}$ was defined as the time duration from the start to the deepest point of the countermovement (velocity is zero); $t_{\text {con }}$ was the time duration from the bottom of the countermovement to the moment of takeoff. Total time of the jump $\left(t_{\text {total }}\right)$, as described elsewhere [5] is the time duration from the start of the countermovement to takeoff and was calculated by adding $t_{\text {ecc }}$ and $t_{\text {con }}$. The ratio of $t_{\text {ecc }}$ to $t_{\text {total }}$ was calculated and expressed as percentage. Total vertical displacement $\left(\mathrm{Vh}_{\text {total }}\right)$ was calculated by adding vertical displacement during the eccentric and the concentric phases of the jump. Jump height was calculated based on the flight time method $[2,5,14]$. Flight time was calculated from the moment of takeoff to the moment of landing. Reactive Strength Index modified was calculated by dividing jump height by the time to takeoff as described previously [2,5].

Table 1. Descriptive Characteristics of the Subjects by Age Group (mean \pm sd)

\begin{tabular}{|c|c|c|c|c|}
\hline Variable & 15 years $(n=23)$ & 16 years $(n=28)$ & 17 years $(n=36)$ & 18 years $(n=28)$ \\
\hline Age (years) & $15.13 \pm 0.24$ & $16.00 \pm 0.31$ & $16.94 \pm 0.33$ & $17.86 \pm 0.19$ \\
\hline Body mass (kg) & $72.66 \pm 12.56$ & $71.49 \pm 10.03$ & $76.40 \pm 8.83$ & $74.52 \pm 8.68$ \\
\hline Body height (m) & $1.81 \pm 0.08$ & $1.80 \pm 0.08$ & $1.83 \pm 0.08$ & $1.83 \pm 0.08$ \\
\hline Training age (years) & $9.02 \pm 1.66$ & $10.09 \pm 1.64$ & $10.35 \pm 2.40$ & $10.96 \pm 2.54$ \\
\hline Training load (hrs/week) & $9.41 \pm 2.30$ & $11.01 \pm 2.89$ & $11.82 \pm 2.72$ & $12.30 \pm 3.59$ \\
\hline
\end{tabular}

$\S=\mathrm{p}<0.05$ statistically significant difference compared to 16 -year-old age group; $*=\mathrm{p}<0.05$ statistically significant difference compared to 17 -year-old age group; $\#=\mathrm{p}<0.05$ statistically significant difference compared to 18 -year-old age group.

Table 2. Descriptive Characteristics of the Subjects by Sport Discipline (mean \pm sd)

\begin{tabular}{|c|c|c|c|c|}
\hline Variable & $\operatorname{soccer}(n=58)$ & basketball $(n=18)$ & handball $(n=24)$ & rowing $(n=15)$ \\
\hline Age (years) & $16.73 \pm 0.93$ & $16.75 \pm 0.91$ & $15.61 \pm 0.86$ & $17.31 \pm 0.47$ \\
\hline Body mass (kg) & $68.55 \pm 6.69$ & $80.99 \pm 7.41$ & $77.85 \pm 12.88$ & $80.48 \pm 5.96$ \\
\hline Body height (m) & $1.77 \pm 0.06$ & $1.93 \pm 0.05$ & $1.82 \pm 0.05$ & $1.85 \pm 0.03$ \\
\hline Training age (years) & $10.83 \pm 1.99$ & $10.09 \pm 1.64$ & $9.05 \pm 1.63$ & $9.21 \pm 2.98$ \\
\hline Training load (hrs/week) & $11.16 \pm 3.01$ & $12.63 \pm 3.55$ & $9.94 \pm 2.41$ & $12.10 \pm 2.75$ \\
\hline
\end{tabular}

$\S=$ statistically significant difference compared to soccer; $*=\mathrm{p}<0.05$ statistically significant difference compared to handball; \#= $<0.05$ statistically significant difference compared to basketball. 


\subsection{Procedures}

The measurements were organized within the typical performance monitoring program of participants and took place between September 2015 to April 2016. All participants were tested by the same examiners. This study was approved by the University's Research Ethics Committee.

\subsection{Statistical Analyses}

All values are expressed as means \pm s.d. Reliability was measured using intraclass correlation coefficient (ICC). The Kolmogorov-Smirnov test was used to test the normality of the data, and Levene's test was used to test the homogeneity of variance. Comparison of means between the different age-groups and sport disciplines was performed using two-way analysis of variance. If a significance $F$ value was recorded, we used Scheffe post hoc test to look for inter-group differences. Effect sizes were tested using eta squared; an effect size of 0.01 was accepted as small, 0.06 as medium and 0.14 as large as described previously [15]. Pearson product-moment correlation was performed to examine the relationship between all variables. Correlation coefficients were classified as 0.0-0.1 (trivial); 0.1-0.3 (small); 0.3-0.5 (moderate); 0.5-0.7 (large); 0.7-0.9 (very large); 0.9-1.0 (near perfect) [16]. Linear regression analysis was used to assess the predictive power of the average eccentric force, average concentric force and total vertical displacement as the independent variables in RSImod as the dependent variable. Statistical significance was set at $p<.05$. SPSS 19.0 (IBM, New York, NY, USA) was used for the statistical analysis.

\section{Results}

Mean value of the ICC was 0.78 for single measures (95\% CI $=0.72-0.83$ ). Distribution of data was found not to differ from normal. Levene's test indicated equal variances for jump height, RSImod, Fecc, Fcon, ttotal and tcon, whereas unequal variances were found for tecc and Vhtotal. A 4x4 two-way ANOVA with age group (four categories: 15 to 18 years) and sport groups (four categories: soccer, basketball, handball, rowing) as independent factors revealed a significant main effect for sport group (F3,115=11.38; $\eta 2=0.25$; $\mathrm{p}<.01$ ), with soccer and basketball players demonstrating higher values than handball players and rowing, where there was no significant effect for age group (F $3,115=0.64 ; \eta 2=0.01 ; p>.05)$. There was no interaction between sport groups and age groups ( $\mathrm{F}$ $7,115=0.25 ; \eta 2=0.01 ; p>.05)$. Among the extracted variables from the force time curve, there was a significant main effect for sport discipline in average eccentric force ( $F$ 3,115 $=5.11 ; \eta 2=0.13 ; \mathrm{p}<.01$ ) and in average concentric force $(F 3,115=6.22 ; \eta 2=0.15 ; p<.01)$, with basketball players producing, in both variables, higher force compared to the other sport groups. Also, there was a significant main effect for ttotal ( $F$ 3,115=12.05; $\eta 2=0.26 ; \mathrm{p}<.01$ ), with soccer and basketball players having a shorter time duration than handball players and rowing. Descriptive data of countermovement jump by age group and by sport discipline are presented in Table 3 and Table 4.

Table 3. Descriptive Data of Countermovement Jump by Age Group (mean \pm sd)

\begin{tabular}{|c|c|c|c|c|}
\hline \multirow[b]{2}{*}{ Variable } & \multicolumn{4}{|c|}{ Age group (years) } \\
\hline & 15 & 16 & 17 & 18 \\
\hline Jump Height (m) & $0.32 \pm 0.04 * \#$ & $0.34 \pm 0.06$ & $0.36 \pm 0.04$ & $0.37 \pm 0.05$ \\
\hline $\operatorname{RSImod}(\mathbf{m} / \mathbf{s})$ & $0.40 \pm 0.07$ & $0.43 \pm 0.11$ & $0.45 \pm 0.11$ & $0.46 \pm 0.12$ \\
\hline$t_{\text {ecc }}(s)$ & $0.51 \pm 0.07$ & $0.51 \pm 0.09$ & $0.54 \pm 0.15$ & $0.54 \pm 0.13$ \\
\hline$t_{\text {con }}(s)$ & $0.31 \pm 0.04$ & $0.30 \pm 0.04$ & $0.29 \pm 0.03$ & $0.30 \pm 0.04$ \\
\hline$t_{\text {total }}(s)$ & $0.82 \pm 0.09$ & $0.81 \pm 0.13$ & $0.84 \pm 0.18$ & $0.85 \pm 0.17$ \\
\hline$t_{\text {ecc }}: t_{\text {total }}(\%)$ & $62.21 \pm 3.58$ & $62.67 \pm 2.70$ & $64.23 \pm 3.71$ & $63.66 \pm 2.55$ \\
\hline $\mathbf{F}_{\text {ecc }}(\mathbf{N})$ & $512 \pm 109$ & $564 \pm 129$ & $582 \pm 198$ & $566 \pm 194$ \\
\hline$F_{\text {con }}(\mathbf{N})$ & $585 \pm 104 * \#$ & $636 \pm 160$ & $683 \pm 111$ & $688 \pm 166$ \\
\hline$V h_{\text {total }}(\mathbf{m})$ & $0.84 \pm 0.10$ & $0.85 \pm 0.15$ & $0.84 \pm 0.11$ & $0.88 \pm 0.12$ \\
\hline
\end{tabular}

RSImod $=$ Reactive Strength Index modified; $t_{\text {ccc }}=$ time during the eccentric phase; $t_{\text {con }}=$ time during the concentric phase; $t_{\text {total }}=$ time from the start of the countermovement to takeoff; $t_{\text {ecc }}: t_{\text {total }}=$ ratio of the eccentric time to total time; $F_{\text {ecc }}=$ average force during the eccentric phase; $F_{\text {con }}=$ average force during the concentric phase; $\mathrm{Vh}_{\text {total }}=$ vertical displacement from the start of the countermovement to takeoff.

$*=p<0.05$ statistically significant difference compared to 17 -year-old age group; $\#=p<0.05$ statistically significant difference compared to 18 -year-old age group.

Table 4. Descriptive Data of Countermovement Jump by Sport Discipline (mean \pm sd)

\begin{tabular}{|c|c|c|c|c|}
\hline Variable & $\operatorname{soccer}(n=58)$ & basketball $(n=18)$ & handball $(n=24)$ & rowing $(n=15)$ \\
\hline Jump Height (m) & $0.36 \pm 0.05$ & $0.37 \pm 0.05$ & $0.32 \pm 0.05$ & $0.34 \pm 0.04$ \\
\hline $\operatorname{RSImod}(\mathrm{m} / \mathrm{s})$ & $0.47 \pm 0.09 * \#$ & $0.49 \pm 0.10 * \#$ & $0.36 \pm 0.07$ & $0.35 \pm 0.08$ \\
\hline$t_{\text {ecc }}(s)$ & $0.50 \pm 0.09$ & $0.46 \pm 0.04$ & $0.57 \pm 0.10$ & $0.66 \pm 0.18$ \\
\hline$t_{\operatorname{con}}(s)$ & $0.29 \pm 0.03$ & $0.28 \pm 0.03$ & $0.33 \pm 0.04$ & $0.33 \pm 0.02$ \\
\hline$t_{\text {total }}(s)$ & $0.79 \pm 0.01 * \#$ & $0.74 \pm 0.74 * \#$ & $0.91 \pm 0.13$ & $0.99 \pm 0.20$ \\
\hline $\mathbf{t}_{\text {ecc }}: \mathbf{t}_{\text {total }}(\%)$ & $63.09 \pm 2.45$ & $61.90 \pm 1.78$ & $63.45 \pm 3.91$ & $65.63 \pm 4.94$ \\
\hline$F_{\text {ecc }}(\mathbf{N})$ & $552 \pm 165$ & $706 \pm 152 \S * \#$ & $505 \pm 129$ & $503 \pm 157$ \\
\hline$F_{\text {con }}(N)$ & $657 \pm 148$ & $764 \pm 130 \S * \#$ & $584 \pm 115$ & $615 \pm 79$ \\
\hline$V h_{\text {total }}(\mathbf{m})$ & $0.82 \pm 0.11$ & $0.83 \pm 0.09$ & $0.89 \pm 0.13$ & $0.93 \pm 0.05$ \\
\hline
\end{tabular}

RSImod $=$ Reactive Strength Index modified; $t_{\text {ecc }}=$ time during the eccentric phase; $t_{\text {con }}=$ time during the concentric phase; $t_{\text {total }}=$ time from the start of the countermovement to takeoff; $t_{\text {ecc }}: t_{\text {total }}=$ ratio of the eccentric time to total time; $F_{\text {ecc }}=$ average force during the eccentric phase; $F_{\text {con }}=$ average force during the concentric phase; $\mathrm{Vh}_{\text {total }}=$ vertical displacement from the start of the countermovement to takeoff.

$\S=$ statistically significant difference compared to soccer; $*=p<0.05$ statistically significant difference compared to handball; \#= $<<0.05$ statistically significant difference compared to rowing. 
Table 5. Correlation Matrix between the Examined Variables

\begin{tabular}{|c|c|c|c|c|c|c|c|c|c|}
\hline & JH & RSImod & $t_{\text {ecc }}$ & $t_{\text {con }}$ & $t_{\text {total }}$ & $t_{\text {ecc }}: t_{\text {total }}$ & $\mathbf{F}_{\text {ecc }}$ & $\mathbf{F}_{\text {con }}$ & $V h_{\text {total }}$ \\
\hline JH & 1 & & & & & & & & \\
\hline RSImod & $0.76^{* *}$ & 1 & & & & & & & \\
\hline $\mathbf{t}_{\text {ecc }}$ & -0.18 & $-0.69 * *$ & 1 & & & & & & \\
\hline $\mathbf{t}_{\mathrm{con}}$ & $-0.33 * *$ & $-0.76 * *$ & $0.66 * *$ & 1 & & & & & \\
\hline $\mathbf{t}_{\text {total }}$ & $-0.23 *$ & $-0.76 * *$ & $0.98 * *$ & $0.80 * *$ & 1 & & & & \\
\hline $\mathbf{t}_{\text {ecc }}: \mathbf{t}_{\text {total }}$ & 0.09 & $-0.27 * *$ & $0.75 * *$ & 0.04 & $0.61 * *$ & 1 & & & \\
\hline $\mathbf{F}_{\text {ecc }}$ & $0.22 *$ & $0.57 * *$ & $-0.62 * *$ & $-0.55 * *$ & $-0.64 * *$ & $-0.38 * *$ & 1 & & \\
\hline$F_{\text {con }}$ & $0.39 * *$ & $0.50 * *$ & $-0.27 * *$ & $-0.55^{* *}$ & $-0.37 * *$ & 0.13 & $0.44 * *$ & 1 & \\
\hline$V \mathbf{h}_{\text {total }}$ & 0.13 & $-0.40 * *$ & $0.55 * *$ & $0.78 * *$ & $0.65 * *$ & 0.10 & $-0.25^{* *}$ & $-0.38 * *$ & 1 \\
\hline
\end{tabular}

$\mathrm{JH}=$ Jump height; RSImod= Reactive Strength Index modified; $\mathrm{t}_{\mathrm{ecc}}=$ time during the eccentric phase; $\mathrm{t}_{\mathrm{con}}=$ time during the concentric phase; $\mathrm{t}_{\mathrm{total}}=$ time from the start of the countermovement to takeoff; $t_{\text {ecc }}: t_{\text {total }}=$ ratio of the eccentric time to total time; $F_{\text {ecc }}=$ average force during the eccentric phase; $\mathrm{F}_{\mathrm{con}}=$ average force during the concentric phase; $\mathrm{Vh}_{\text {total }}=$ vertical displacement from the start of the countermovement to takeoff. $*=\mathrm{p}<0.05 ; * *=\mathrm{p}<0.01$.

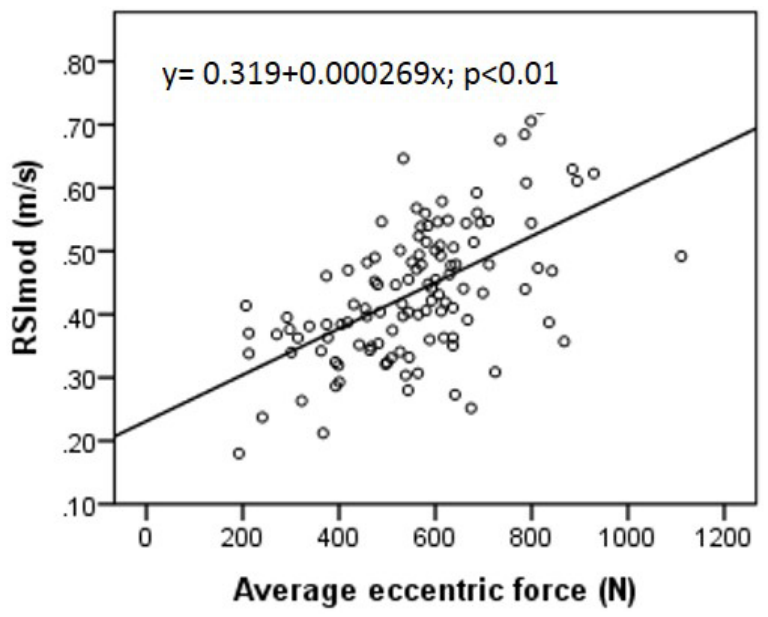

Figure 1. Linear regression between RSImod and average eccentric force

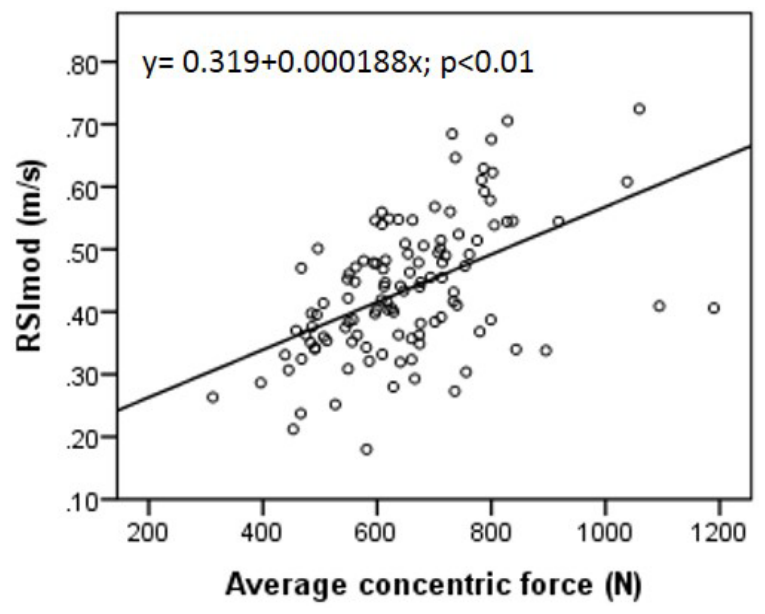

Figure 2. Linear regression between RSImod and average concentric force

Results from the regression analysis are presented in Figure 1 to Figure 3 for the average eccentric force, the average concentric force and the total vertical displacement, respectively. A significant regression equation was found $\left(F_{3,114}=28.87 ; p<.01\right)$ with an $\mathrm{R}^{2}=0.44$. All three independent variables contributed significantly to the total variance of the dependent variable; eccentric and concentric force had a positive prediction $(b=.00024$, $t=4.60, p<.001$ and $b=.000189, t=2.98, p<.01$ respectively), while total vertical displacement had a negative prediction $(b=-.146, t=-2.00, p<.05)$ in RSImod.

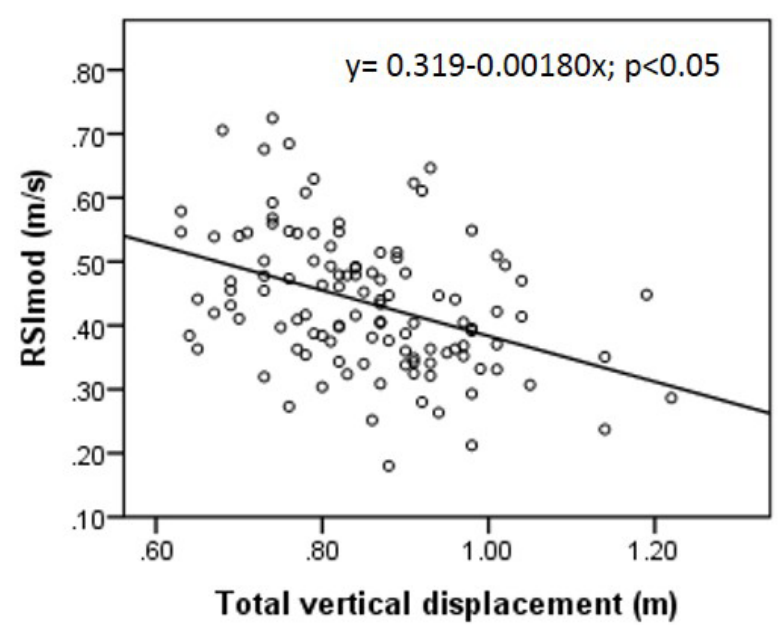

Figure 3. Linear regression between RSImod and total vertical displacement

\section{Discussion}

In this cross-sectional study we examined RSImod in adolescent athletes from different sport disciplines. The main idea was to investigate whether RSImod is a suitable method for the assessment and evaluation of explosiveness in young athletes and if any differences can be detected through growth and athletic development in the age period between 15-18 years. It was also our aim to evaluate whether RSImod differs between athletes from different sport disciplines and to examine any relationships between RSImod and eccentric and concentric force production as well as vertical displacement.

Mean value of intraclass correlation coefficient for RSImod represents excellent reliability $(>0.75)$ based on the classification introduced by Fleiss [17]; however, ICC values in this study were lower than those reported with adult athletes in previous studies: $\mathrm{ICC}=0.85$ for single measures [2] or ICC $=0.96$ [5]), which suggests greater trial-to-trial variability in the movement pattern during CMJ for adolescent athletes.

Mean values of RSImod by age group ranged from 0.39 (15 years old) to 0.46 (18 years old). Reference [5] measured collegiate athletes from various sports and reported RSImod values of $0.41 \pm 0.09$ for men, which is similar to the average values of this study, while in another study [2] average RSImod values of $0.74 \pm 0.19$ for 
young adults were reported. In this latter study, however, subjects participated in plyometric training as part of their annual training program assuming a well-developed explosive ability.

We used a two-way analysis of variance to examine differences in RSImod between age groups and sport groups. The results revealed no interaction between main effects, which can be explained by the fact that age seemed not to have any effect on RSImod. A significant main effect was found for sport discipline, with soccer and basketball players in our sample demonstrating higher values than handball players and rowers. In previous research RSImod was found to vary between exercises of different explosiveness profiles, in particular between athletes of different playing positions in baseball [6], or between plyometric exercises of different intensity [2]. Higher explosiveness for soccer and basketball players, as indicated by RSImod results, was realized in both larger jumps and a shorter time to takeoff, which in the case of basketball players is in accordance with the physical demands of the game. Vertical jumps are one of the most common movements performed by basketball players in trainings and games, and this naturally results in improving their jumping ability [18]. Nevertheless, the results here should be treated carefully; a limitation of the statistical analysis was the unequal sample sizes of the sport groups. In regards to handball players, mean age must also be taken into consideration; handball players were a little more than one year younger than basketball and soccer players. In the case of rowers, a clearly marked difference in RSImod can be seen, most probably due to their endurance-based type of training; explosiveness in rowers seems to be less developed, and this is well demonstrated by RSImod.

We did not find a significant main effect for age groups. Through the examined age period an increasing tendency can be seen between consecutive age groups, but these differences were not statistically significant. There is no clear explanation for the lack of differences between the age groups. Reference [19] suggests that the optimal period for a more profound neuromuscular adaptation is during prepuberty; regarding RSI two periods of accelerated development were recognized - one between the ages of 10 and 11 years and one from 12 to 13 years. Additionally, it has been shown [20] that during prepuberty and early puberty plyometric training, in addition to a regular training program, can be beneficial in terms of increased power and improved jumping and sprinting performance in boys and girls. It seems that for those between the ages of 15 and 18 years, neuromuscular adaptation is less intense and lesser development can be achieved in RSImod. However, we still don't know whether a more explosiveness specific training program could improve RSImod values in this age period. Summarizing two-way anova results, it appears, that RSImod is mostly sport-specific and not age related, and that this explosiveness profile is formed at an earlier stage of the athletic career, than the one examined in this study.

Time to takeoff is the timing component of RSImod, and it changes mainly from variations in eccentric time [11]. The results in this study showed great variability in the time variables; time to takeoff had a coefficient of variation $(\mathrm{CV})$ of over $10 \%$ in all age groups. Mean values of the age groups were almost identical, while mean values of the sport groups differed; soccer and basketball players had shorter time duration than handball players. High variability together with the nature of differences between sub-groups suggest that the participants followed different jumping patterns in their temporal structure and differed by sport and not by age, and this seems to have only a small connection to the height of the jump (Table 5).

Time to takeoff ranged between $0.81 \mathrm{~s}$ to $0.85 \mathrm{~s}$, which is similar to that reported elsewhere $(0.85 \mathrm{~s})$ [5]. On the contrary timing variables here differed from that described in previous research. Reference [11] in their study with well-trained, adult athletes competing in various sports, reported mean values of eccentric time for men of $0.22 \mathrm{~s}$, and a ratio of eccentric to total time of $42 \%$. In our study the lowest mean eccentric time was $0.46 \mathrm{~s}$ for basketball players, while the eccentric to total time ratio was $>60 \%$ in all subgroups, illustrating slower downward movement, which may be indicative of significant potentials for further development in our sample.

Regression analysis showed that force production, both eccentric and concentric, as well as vertical displacement have a significant prediction in RSImod, explaining 42\% of the total variance in RSImod. The strongest predictive power was found for average eccentric force, which clearly supports the role of the latter in explosiveness. Larger eccentric force was associated with higher values in RSImod. High eccentric force enhances overall force production, which in turn can increase jump height, while contributing to decreased time to takeoff through a more rapid countermovement. Based on the correlation coefficients (Table 5), average eccentric force had a negative correlation with the eccentric time $(\mathrm{r}=-0.62)$; larger eccentric force results in shorter time duration during the eccentric phase. Moreover, average eccentric force had a stronger correlation with the timing variables, especially with the eccentric time, than with jump height, suggesting a more profound role for eccentric force in performing a rapid countermovement than in increasing jump height.

A limitation of RSImod is that it includes only the timing component of the jump [7] and does not take into consideration the magnitude of the countermovement, which affects the vertical displacement of the center of mass. Two athletes with the same duration in the time to takeoff and same RSImod values may differ in the total distance they cover during the countermovement and the push off phases, which may refer to differences in explosiveness; the one who covers a larger distance develops larger mean velocity. Vertical displacement had a negative correlation with RSImod; larger vertical displacement results in smaller values in RSImod, suggesting lower explosive ability. However, the large positive correlation of vertical displacement with the time to takeoff indicates, that shorter time to takeoff is associated with a smaller countermovement depth and not necessarily with a faster execution of the jumping movement. Accordingly, in the evaluation of explosiveness besides time to takeoff, we find it useful to consider also the magnitude of the countermovement. 


\section{Conclusions}

In conclusion, RSImod can differentiate between athletes from sports of quite different profiles (endurance-based versus ball games). On the other hand, the lack of differences between the examined age groups indicates that the explosiveness profile, based on RSImod measurements, of adolescent athletes is formed at an earlier stage of their athletic career. The significant relationship between eccentric force and RSImod supports the importance of eccentric force in explosiveness, mainly in the performance of a more rapid countermovement.

\section{References}

[1] Flanagan, E.P. and Comyns, T.M. "The Use of Contact Time and the Reactive Strength Index to Optimize Fast Stretch-Shortening Cycle Training," Strength and Conditioning Journal, 30(6), 32-38, 2008.

[2] Ebben, W.P. and Petushek, E.J. "Using the reactive strength index modified to evaluate plyometric performance," Journal of Strength and Conditioning Research, 24(8), 1983-1987, 2010.

[3] Flanagan, E.P., Ebben, W.P. and Jensen, R.L. "Reliability of the Reactive Strength Index and Time to Stabilization During Depth Jumps," Journal of Strength and Conditioning Research, 22(5), 1677-1682, 2008

[4] Markwick, W. J., Bird, S. P., Tufano, J. J., Seitz, L. B. and Haff, G. G. "The Intraday Reliability of the Reactive Strength Index Calculated From a Drop Jump in Professional Men's Basketball," International Journal of Sports Physiology and Performance, 10: 482-488, 2015.

[5] Suchomel, T.J., Bailey, C.A., Sole, C.J., Grazer, J.L. and Beckham, G.K. "Using Reactive Strength Index-Modified as an Explosive Performance Measurement Tool in Division I Athletes," Journal of Strength and Conditioning Research, 29(4), 899-904, 2015.

[6] Bailey, C.A., Suchomel, T.J., Beckham, G.K., Sole, C.J. and Grazer, J.L. "A comparison of baseball positional differences with reactive strength index-modified," in XXXII International Conference of Biomechanics in Sports, International Society of Biomechanics in Sports, 562-565.

[7] Kipp, K., Kiely, M.T. and Geiser, C.F. "Reactive Strength IndexModified is a Valid Measure of Explosiveness in Collegiate Female Volleyball Players," Journal of Strength and Conditioning Research, 30(5), 1341-1347, 2015.
[8] Aragón-Vargas, L.F. and Gross, M.M. "Kinesiological Factors in Vertical Jump Performance: Difference Among Individuals," Journal of Applied Biomechanics, 13, 24-44, 1997.

[9] Baker, D.G. "10-Year Changes in Upper Body Strength and Power in Elite Professional Rugby League Players - The Effect of Training Age, Stage, and Content," Journal of Strength and Conditioning Research, 27(2), 285-292, 2013.

[10] Peterson, M.D., Alvar, B.A. and Rhea, M.R. "The Contribution of Maximal Force Production to Explosive Movement Among Young Collegiate Athletes," Journal of Strength and Conditioning Research, 20(4), 867-873, 2006.

[11] Laffaye, G., Wagner, P. and Tombleson, T. "Countermovement Jump Height: Gender and Sport-Specific Differences in the ForceTime Variables," Journal of Strength and Conditioning Research, 0(0), 1-10, 2013.

[12] Riggs, M.P. and Sheppard, J.M. "The relative importance of strength and power qualities to vertical jump height of elite beach volleyball players during the counter-movement and squat jump," Journal of Human Sport and Exercise, 4(3), 221-236, 2009.

[13] Martin, R., and Saller, K, Lehrbuch der anthropologie. Fischer Stuttgart, 1957. Fleiss, J.L, The Design and Analysis of Clinical Experiments. John Wiley and Sons, New York, 1986.

[14] Linthorne, N.P, ,Analysis of standing vertical jump using a force platform," American Journal of Physics, 69(11), 1198-1204, 2001.

[15] Lakens, D, „Calculating and reporting effect sizes to facilitate cumulative science: a practical primer for t-test and ANOVAs," Frontiers in Psychology, 4, 1-12, 2013.

[16] Hopkins, W.G. "A scale of Magnitudes for Effect Statistics," Available: http://www.sportsci.org/resource/stats/ [Accessed Oct. 15, 2016].

[17] Fleiss, J.L. The Design and Analysis of Clinical Experiments. John Wiley and Sons, New York, 1986Lloyd, R.S., Oliver, J.L., Hughes, M. G. and Williams, C. A, ,The influence of chronological age on periods of accelerated adaptation of stretch-shortening cycle performance in pre- and post-pubescent boys," Journal of Strength and Conditioning Research, 25(7), 1889-97, 2011.

[18] Ziv, G. and Lidor, R, Vertical jump in female and male basketball players - A review of observational and experimental studies. Journal of Science and Medicine in Sport, 13, 332 -339, 2010.

[19] Lloyd, R.S., Oliver, J.L., Hughes, M. G. and Williams, C. A. "The influence of chronological age on periods of accelerated adaptation of stretch-shortening cycle performance in pre- and post-pubescent boys," Journal of Strength and Conditioning Research, 25(7), 1889-97, 2011.

[20] Chelly, M.S., Hermassi, S. and Shephard, R.J. "Effects of InSeaason Short-term Plyometric Training Program on Sprint and Jump Performance on Young Male Track Athletes," Journal of Strength and Conditioning Research, 29(8), 2128-2136, 2015. 